\title{
Deposición de Hidrogeno a Subpotencial sobre Oro Policristalino y con Orientación Preferida
}

\author{
Maria G. Sustersic, Thelma A. Zanón, Sonia G. Albano y Alicia E. Von Mengershausen \\ Universidad Nacional de San Luís, Facultad de Ingeniería y Ciencias Económico Sociales, \\ Avda. 25 de Mayo № 384, 5730 Villa Mercedes, San Luís-Argentina \\ (e-mail: masus@fices.unsl.edu.ar)
}

\begin{abstract}
Resumen
Se estudia la deposición de hidrógeno a potenciales menores que los correspondientes a la reacción de evolución de hidrógeno sobre oro policristalino cuya superficie ha sido preferentemente orientada según los tres planos de bajo índice. Varios procesos contribuyen a las corrientes anódica y catódica en la región de potenciales de carga de doble capa: la adsorción específica de aniones y su desorción, el levantamiento de la reconstrucción superficial, la oxidación del hidrógeno y la deposición de hidrógeno a subpotencial. Las alturas relativas de las distintas contribuciones de corriente cambian con la orientación preferencial. Los máximos correspondientes a la oxidación del hidrógeno depositado crecen continuamente mientras que aquéllos que corresponden al levantamiento de la reconstrucción superficial alcanzan una altura límite. Se concluye que es posible distinguir las contribuciones farádicas de aquéllas de origen capacitivo en la región de potenciales correspondiente a la carga de la doble capa en una solución de ácido sulfúrico $0.5 \mathrm{M}$.
\end{abstract}

\section{Hydrogen underpotential deposition on preferentially oriented polycrystalline gold}

\begin{abstract}
Hydrogen deposition at lower potentials than those corresponding to the hydrogen evolution reaction on polycrystalline gold whose surface has been preferentially oriented according to the three low index planes, has been studied. Several processes contribute to the anodic and cathodic currents at the double layer charging potential region: the specific anion adsorption and desorption, the lift of surface reconstruction, the hydrogen oxidation and the hydrogen underpotential deposition. The relative heights of different current contributions change with the surface preferential orientation. The hydrogen oxidation peaks grow continuously while those corresponding to the lift of surface reconstruction reach a limited height. These facts allow distinguishing the faradic current contributions from the capacitive current contributions at the double layer charging potentials in $0.5 \mathrm{M}$ sulphuric acid solution.
\end{abstract}

Keywords: hydrogen deposition, gold electrodes, surface reconstruction, electric double layer 


\section{INTRODUCCION}

La reacción de evolución de H ocurre por medio de una etapa de descarga (Schmid, 1967), en la cual el átomo se adsorbe sobre la superficie del metal:

$\mathrm{H}^{+}+\mathrm{e} \leftrightarrow \mathrm{H}_{\text {ads }}$

seguida de una recombinación, que puede ser química o electroquímica, dependiendo del metal, de su historia previa y del pretratamiento. Sobre oro, parte del H atómico producido en la reacción (1) se recombina y parte difunde hacia el interior del metal (Martins et al., 1987) mediante difusión transgranular (Chao et al., 1980). Estos autores utilizaron sobrepotenciales catódicos para producir $\mathrm{H}$ en gran cantidad. En la región de potenciales donde no se observa desprendimiento de $\mathrm{H}$ (Sustersic et al., 2004), los átomos también difunden hacia adentro. La presencia de $\mathrm{H}$ ha sido demostrada en estas condiciones por reducción del ion $\mathrm{Ag}^{+}$seguida de "stripping" de la capa de plata depositada. Cuando la concentración de $\mathrm{H}$ en el interior cercano a la superficie es alta, la difusión hacia adentro se ve impedida y se favorece la adsorción.

Los voltamperogramas cíclicos del oro policristalino en la región de potenciales de carga de doble capa hasta el momento no han sido obtenidos con escalas suficientemente sensibles como para ver la estructura fina de las relaciones corriente/potencial. Solamente cuando se trabajó con monocristales se utilizaron escalas suficientemente sensibles (Kolb, 1993; Kolb, 1996; Schneider y Kolb, 1988; Shi et al. 1995). El objetivo de estos trabajos con mono-cristales ha sido encontrar los picos correspondientes al levantamiento de la reconstrucción superficial (LSR). Las superficies reconstruidas se obtenían con tratamientos térmicos en ultra alto vacío o cargando al electrodo negativamente. Este tipo de tratamiento se denominó "reconstrucción asistida por el potencial (PASR)". No se mencionó, en la literatura, la deposición de $\mathrm{H}$ a subpotencial sobre oro policristalino ni tampoco sobre monocristales. Es precisamente en la región de potenciales de carga de doble capa donde se manifiesta el sistema redox del $\mathrm{H}$ cuyas corrientes farádicas se superponen a las corrientes capacitivas de carga de doble capa y se distingue de aquéllas originadas por el LSR. La capacidad del oro para absorber H (Córdova Orellana et al., 1979; Martins et al. 1987; Chao et al. 1980) facilita la diferenciación, porque los picos de corriente de oxidación de $\mathrm{H}$ crecen continuamente con el tiempo de carga del $\mathrm{H}, \mathrm{t}$, mientras que los picos de corriente del (LSR) alcanzan una altura límite para un tiempo al cual la reconstrucción es completa. Manteniendo el electrodo a un potencial negativo con respecto al potencial de carga cero $(\mathrm{pcc})$, los diferentes planos cristalinos se reconstruyen. Simultáneamente, se produce $\mathrm{H}$ mediante la reacción (1).

El sobrepotencial de la reacción de evolución de $\mathrm{H}$ es menor sobre la superficie policristalina que sobre una monocristalina. Por este motivo, no es posible someter al electrodo policristalino a un potencial negativo sin que se deposite $\mathrm{H}$ sobre el mismo. Esta interferencia no aparece con los monocristales, por cuya causa, en las investigaciones con monocristales la deposición de hidrógeno pasó desapercibida. Los electrodos que se utilizan en electrocatálisis son policristalinos. Esto es, consisten en granos que son truncados en la superficie mostrando cada uno de ellos una orientación diferente. Esto, y la presencia de bordes de grano son las características más destacadas de los metales policristalinos. Cerca del borde la densidad de imperfecciones es mayor que dentro del grano, característica ausente en los monocristales. Evidentemente, es esta la causa del efecto catalítico que ejerce la superficie policristalina sobre la reducción del ion $\mathrm{H}^{+}$. Si el electrodo se somete a una onda de potencial cuadrada de alta frecuencia (Perdriel et al., 1986) se puede obtener una orientación preferencial de los átomos en la superficie según uno de los planos. La amplitud y la frecuencia de la onda definen el tipo de orientación obtenida. Sin embargo, la orientación preferencial no elimina los bordes de grano. Por este motivo, la deposición de hidrógeno y la reconstrucción superficial de los distintos granos ocurren simultáneamente también sobre los electrodos con orientación preferida. Sobre la superficie monocristalina puede ocurrir la PASR sin cargar al electrodo con hidrógeno. Sin embargo, los estudios con monocristales se llevaron a cabo para comprender mejor los policristales, ya que los electrodos reales son policristalinos. Los metales policristalinos consisten de un ensamblaje de monocristales llamados granos. Muchos estudios se han hecho con electrodos de oro monocristalinos. Sin embargo, no se han relacionado con el comportamiento de los policristales para poder conocer el comportamiento de los sistemas prácticos, ya que los electrodos monocristalinos existen solamente 
en el laboratorio. La orientación superficial preferida es una forma de transición entre el monocristal y el policristal que le da a este un cierto carácter de monocristal sin eliminar el ensamblaje de granos.

Si las corrientes voltamétricas se debieran solamente a la carga de la doble capa eléctrica y la capacidad de esta fuese constante, el voltamperograma resultante de la aplicación de un potencial triangular, debería ser un rectángulo. Las desviaciones se deben a los cambios en la capacidad de la doble capa eléctrica, originados por la adsorción y desorción de aniones, al levantamiento de la reconstrucción superficial y a la superposición de corrientes farádicas que en este caso correspoden al sistema redox del $\mathrm{H}$ a subpotencial. Hasta el momento se creía que la deposición de $\mathrm{H}$ a subpotencial (HUPD) no existía. Rand y Wood mencionaron su existencia, pero en cantidades tan bajas que no merecían atención (Rand y Wood, 1972). Sin embargo, la (HUPD) sobre oro puede ser vista en los registros voltamperométricos (Sustersic et al. 2004) aumentando la sensibilidad de la escala y la velocidad de barrido, v, como se muestra aquí. El $\mathrm{H}$ sobre oro merece atención porque juega un papel importante en reacciones superficiales, por ejemplo aquellas que involucran al $\mathrm{SO}_{2}(\mathrm{Kunning}$ et al. 1986; Von Mengershausen et al., 1989; Sustersic et al, 1989). El SO $\mathrm{SO}_{2}$, por acción del $\mathrm{H}$, se reduce a azufre que presenta actividad electrocatalítica (Zaniolo et al., 1996; Jaram et al., 1978).

\section{MATERIALES Y METODOS}

Se utilizó una celda electroquímica de vidrio pirex de dos compartimentos. Un electrodo reversible de $\mathrm{H}$ en un compartimiento separado del compartimiento central se utilizó como electrodo de referencia. El contra electrodo era una chapa de oro de $9 \mathrm{~cm}^{2}$ de área aparente y el electrodo de trabajo un alambre de oro de $1 \mathrm{~mm}$ de diámetro y $3 \mathrm{~cm}$ de longitud. La pureza del oro para ambos electrodos era de $5 \mathrm{~N}$. Como electrolito se utilizó ácido sulfúrico $0,5 \mathrm{M}$, preparado con droga analítica y agua tridestilada. La celda se desgasificaba con burbujeo de nitrógeno ultra puro. El electrodo de oro policristalino se trataba como se describe previamente (Sustersic, et al., 2002) y luego se sometía a una onda cuadrada de potencial de alta frecuencia para obtener una determinada orientación preferida (Perdriel et al. 1986). Para esto se utilizaba un generador de onda cuadrada programable conectado a un potenciostato ambos marca LYP. La señal se observaba en la pantalla de un osciloscopio digital marca Nicolet. Las experiencias se llevaban a cabo a temperatura ambiente. El oro se cargaba con $\mathrm{H}$ mediante electrólisis a $0,1 \mathrm{~V}$. La cantidad de $\mathrm{H}$ cargado se controlaba mediante el tiempo de electrólisis, t. El burbujeo continuo con nitrógeno ultra puro y la hermeticidad de la celda evitaban la contaminación del electrolito durante los tiempos de electrólisis largos. Las experiencias de voltametría cíclica se llevaron a cabo con un generador de función rampa conectado a un potenciostato ambos marca LYP. La respuesta corriente/potencial se registraba con un registrador X-Y marca ALLEN.

La orientación preferencial del electrodo de oro se controlaba con el perfil potenciodinámico en la región de potenciales de formación electroquímica del óxido de oro (Perdriel et al. 1986). Por este motivo, se incluyó, en las figuras 1, 3, 4 y 8 el perfil potencial/corriente completo, obtenido barriendo el potencial entre 0,1 y $1,7 \mathrm{~V}$.

\section{RESULTADOS Y DISCUSIÓN}

En las figuras, los símbolos $I_{a}, I I_{a}, I I I_{a}, I V_{a}, I_{c}, I I_{c}, I I I_{c}$ y $I V_{c}$ son símbolos de las corrientes en distintas regiones de potencial de los voltamperogramas, que pueden ser picos de corriente, ondas o combinación de varias contribuciones de corriente. La figura 1 muestra registros voltamperométricos obtenidos en ácido sulfúrico $0,5 \mathrm{M}$ con oro policristalino sin ninguna orientación preferencial. La figura 1a muestra el voltamperograma obtenido barriendo entre 0,1 y $1,7 \mathrm{~V}$. La figura $1 \mathrm{~b}$ muestra el efecto del tiempo de electrólisis, $t$, a $0,1 \mathrm{~V}$, sobre los voltamperogramas corridos entre 0,1 y $1,2 \mathrm{~V}$ con la misma superficie. Los voltamperogramas de la figura $1 \mathrm{~b}$ se obtuvieron con una escala sensible y por eso se puede observar en ellos la estructura fina de la relación corriente/potencial. En la misma región de potencial, el voltamperograma obtenido en la región de potenciales de carga de doble capa y registrado con escala menos sensible (figura 1a), no muestra ninguna particularidad y aparece como el conjunto de dos líneas paralelas de un rectángulo. Las principales características de la figura $1 \mathrm{~b}$ se describen a continuación: La corriente $I_{a}$ da cuenta, en parte, del cambio de la capacidad en la doble capa originada por la adsorción de aniones (el pcc del oro policristalino es 0,15 V) (Florit et al, 1984). Sin embargo, la corriente decrece con t porque ocurre la PASR simultáneamente con la carga de $\mathrm{H}$. 


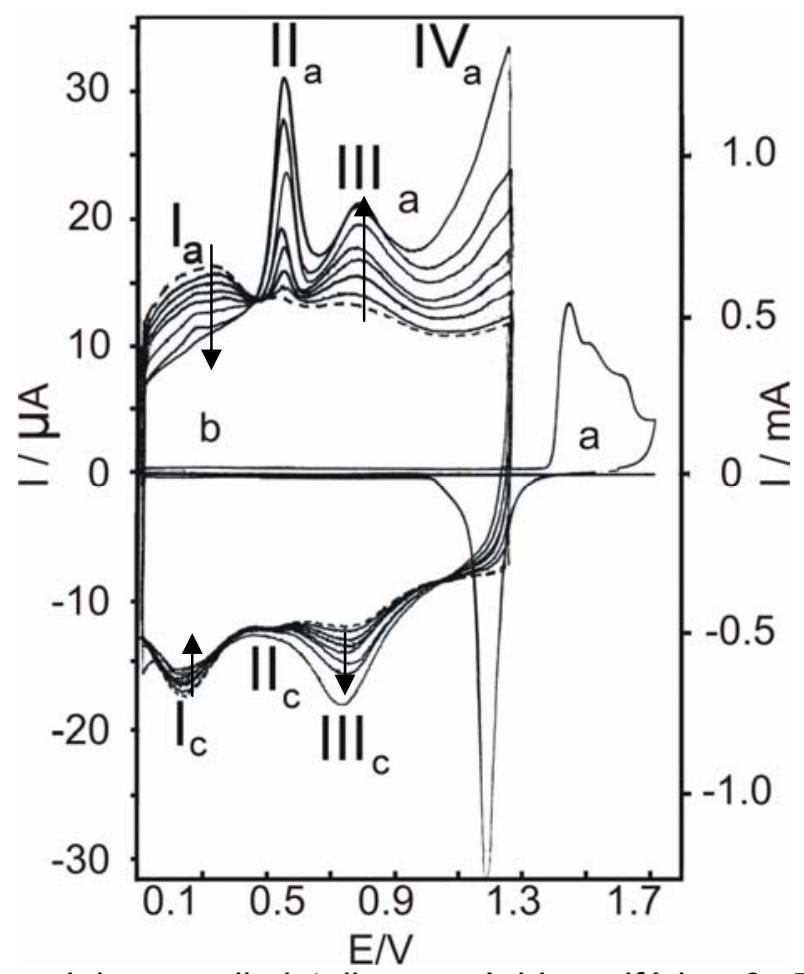

Fig. 1: (a) Voltamperograma del oro policristalino en ácido sulfúrico $0,5 \mathrm{M}$ barriendo en dirección anódica entre 0,1 y 1,7 V. (b) Influencia del tiempo de electrólisis en $0,1 \mathrm{~V}$ sobre los voltamperogramas obtenidos barriendo en dirección anódica entre 0,1 y 1,3 V. $\mathrm{t}=0$ (linea punteada), 6, 20, 40, 60, 120, 180 y 240 min para los registros que se alejan del blanco en el sentido señalado por las flechas. La línea punteada, con $\mathrm{t}=0$, corresponde al blanco sin previa electrólisis. $40 \mathrm{mV} / \mathrm{s} ; 25^{\circ} \mathrm{C}$.

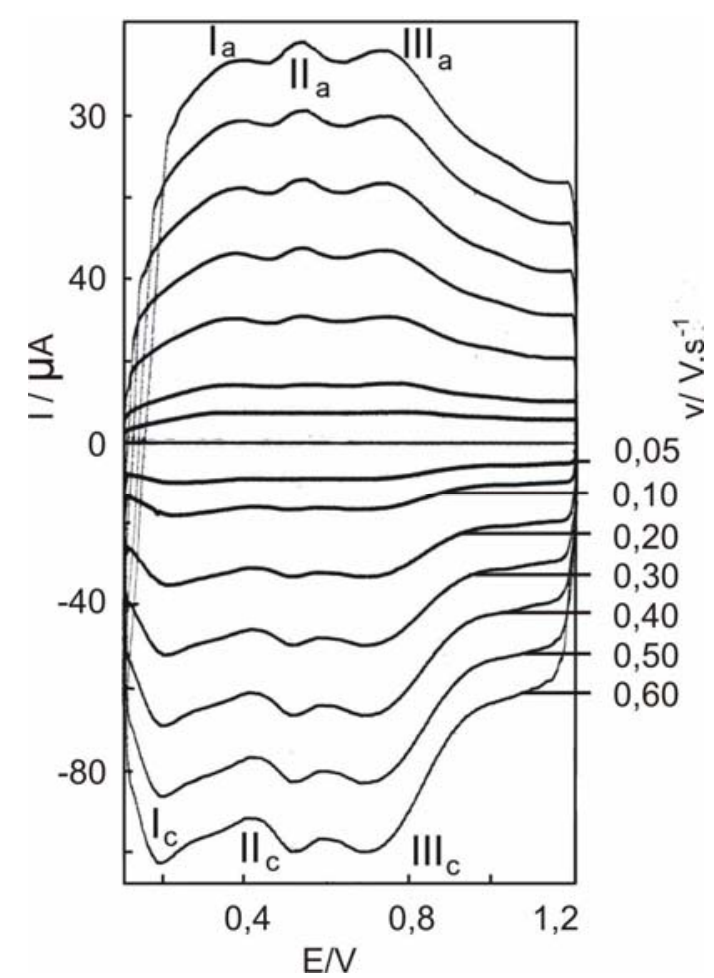

Fig. 2: Voltamperogramas obtenidos con el oro policristalino barriendo en dirección catódica entre 1,2 y $0,1 \mathrm{~V}$ con diferentes velocidades de barrido. $\mathrm{T}=25^{\circ} \mathrm{C}$.

Las superficies $(100)$ y $(111)$ adquieren estructuras hexagonales y $(\sqrt{3} \times 22)$ respectivamente (Kolb, 1993; Kolb, 1996; Schneider y Kolb, 1988; Shi et al. 1995), que adsorben menos aniones que las estructuras $(1 \times 1)$ por ser estructuras más compactas. La corriente en la región designada $l_{a}$ tiene la contribución de LSR del plano (111) como resulta de la comparación con los voltamperogramas del 
electrodo orientado preferentemente según el plano (111) (Fig. 3) y se discute más adelante. A 0,53 $V$ aparece un pico $I_{a}$, cuya altura crece con t. La fig.1 muestra solamente barridos con tiempos de hasta 240'. En la práctica, se esperaron 12 horas sin alcanzar el pico $/ I_{c}$ una altura límite. En un trabajo previo (Sustersic et al., 2004), se demuestra que este pico se debe a la oxidación de H. Aquí se completa la información mostrando que la oxidación de $\mathrm{H}$ a ese potencial ocurre sobre el plano (111) difundiendo desde el interior del metal, como se discute más adelante. Podría ser posible la adsorción de impurezas de la solución durante tiempos tan largos. Sin embargo, la agudeza del pico y el potencial del máximo no cambian a pesar del tiempo de espera largo. La oxidación de impurezas de la solución, debería producir una deformación del pico, pues su potencial de oxidación es distinto del potencial de oxidación del H. Si la solución tiene trazas de algún contaminante orgánico se observan contribuciones de corriente a la izquierda de $I I_{a}$ cuando se utilizan $t$ largos. Una onda de corriente $I I I_{a}$ crece con $t$, alcanzando un valor límite para $t=180$ '. El potencial de corriente máximo es muy próximo al encontrado (Kolb, 1993; Kolb, 1996; Schneider y Kolb, 1988) para LSR del plano (100) con el monocristal. Se observan dos diferencias principales con respecto al LSR de la superficie monocristalina (100): el tiempo para alcanzar la corriente máxima es mucho más largo (el monocristalino necesita unos pocos minutos) y el pico de corriente tiene la forma de una onda que abarca un amplio dominio de potencial. A potenciales más positivos la corriente $I V_{a}$ crece, mostrando un hombro a 1,2 V. En la contribución de corriente $I V_{a}$, participan los planos menos compactos (100 y 110) como se explica más adelante. El barrido inverso presenta un pico de corriente catódica a 0,75 $\mathrm{V}\left(I I I_{c}\right)$, una onda a $0,5 \mathrm{~V}\left(I I_{c}\right)$ y un pico a $0,25 \mathrm{~V}\left(I_{c}\right)$. El primero crece con t, el segundo no cambia con $\mathrm{t}$, mientras que el tercero disminuye. Para identificar estas contribuciones de corriente catódica, se han realizado barridos en dirección catódica entre 1,2 y 0,1 V (fig. 2) cambiando la velocidad de barrido, $v$. La corriente $I_{c}$, que en la figura 1 es casi imperceptible, se define como pico de corriente para $\mathrm{v}$ mayores que $0,1 \mathrm{v} / \mathrm{seg}$ en la figura 2 . Su potencial es el mismo que el de $\| I_{a}$. Si $I_{a}$ y $I_{c}$ corresponden a la reacción (1) directa e inversa, se trata de una reacción muy reversible, característica de la reacción redox del $\mathrm{H}$. Esto es una prueba más a favor de la H UPD.

La fig. 3 muestra la misma experiencia que la fig.1, pero la superficie ha sido orientada preferentemente (Perdriel et al., 1986) según el plano (111). La corriente $I_{a}$ crece con t y muestra un pico pequeño y angosto a $0,25 \mathrm{~V}$ seguido de una onda chata. Este perfil es típico del LSR: un pico angosto debido al cambio de potencial de carga cero sobre el plano (Kolb, 1993; Kolb, 1996; Schneider y Kolb, 1988; Shi et al. 1995) y una onda chata originada por el cambio de capacidad de la doble capa proveniente del reacomodamiento de los aniones adsorbidos sobre el plano no reconstruido. En elcaso de los monocristales aparece un alto y agudo pico en lugar del pico pequeño y angosto (Shi et al. 1995). El pico de corriente $I_{a}$, crece con el tiempo y es el principal pico del perfil. Como se trata de un electrodo orientado según el plano (111), el número de sitios superficiales con esta orientación es alto. Como el pico $I I_{a}$ es el que presenta corrientes más altas, es muy probable que se deba a la oxidación del $\mathrm{H}$ en el plano (111). Las corrientes $I_{a}$ y $I I I_{a}$ crecen con $t$, en menor parte por su propio crecimiento y en mayor parte por la superposición con la corriente del pico $I I_{a}$. El pequeño y angosto pico en ca. $0,58 \mathrm{~V}$ y la onda chata entre 0,68 y 0,9 V aparecen a potenciales próximos a los LSR del plano (100) del monocristal (Kolb, 1993; Kolb, 1996) Por este motivo se atribuyen a este proceso. La cupla de los picos $I I I^{\prime}$ y y $I I I_{c}$, se obseva también con el monocristal (Shi et al., 1994). Se debe a una multicapa ordenada de sulfato. Como la diferencia de potencial entre $I_{a}$ y $I I I^{\prime}$ es próxima a la del oro (111) monocristalino, la corriente $I_{a}$ se debe probablemente al LSR del plano (111). Mejor dicho, tiene, probablemente, una contribución de ese fenómeno, ya que en primer lugar se adsorben los aniones cuya presencia en la superficie origina el levantamiento de la reconstrucción del plano (111). A $1,2 \mathrm{~V}$ el pico $I V_{a}$ crece con $\mathrm{t}$. La onda $I I I_{c}$ consiste de una onda chata y un de pico delgado y angosto situado casi en los mismos potenciales que los correspondientes a $I I I_{a}$. Parecería ser el proceso inverso de $I I I_{a}$. Sin embargo, de acuerdo con Kolb, el LSR no es un proceso reversible. Para reconstruir de nuevo el plano (100), es necesario mantener el electrodo a un potencial negativo con respecto al pcc, Si embargo, la reconstrucción puede ocurrir durante el barrido catódico en menor extensión que en el caso en que el electrodo se mantiene en un potencial catódico. Los voltamperogramas de Kolb muestran esta posibilidad. Probablemente PASR/LSR ocurre reversiblemente en algunos sitios de la superficie. Cuando se adsorben aniones, la reconstrucción se levanta. Durante el ciclo inverso unos pocos sitios se reconstruyen de nuevo. Una interpretación alternativa del origen de las corrientes en $I I I_{c}$ puede ser la desorción de aniones en la superficie no reconstruída, para dar cuenta de la onda chata y la reducción del óxido de oro hidratado (Vela et al., 1995) para interpretar el pico pequeño y 
delgado. El pico $I_{c}$ aparece al mismo potencial que el $I_{a}$, hecho que pone de manifiesto nuevamente la reversibilidad de la reacción (1). La altura de $I_{c}$ crece ligeramente con t. También $I_{c}$, que corresponde a la desorción de aniones en el plano (111) no reconstruido, muestra una tendencia a crecer con t.

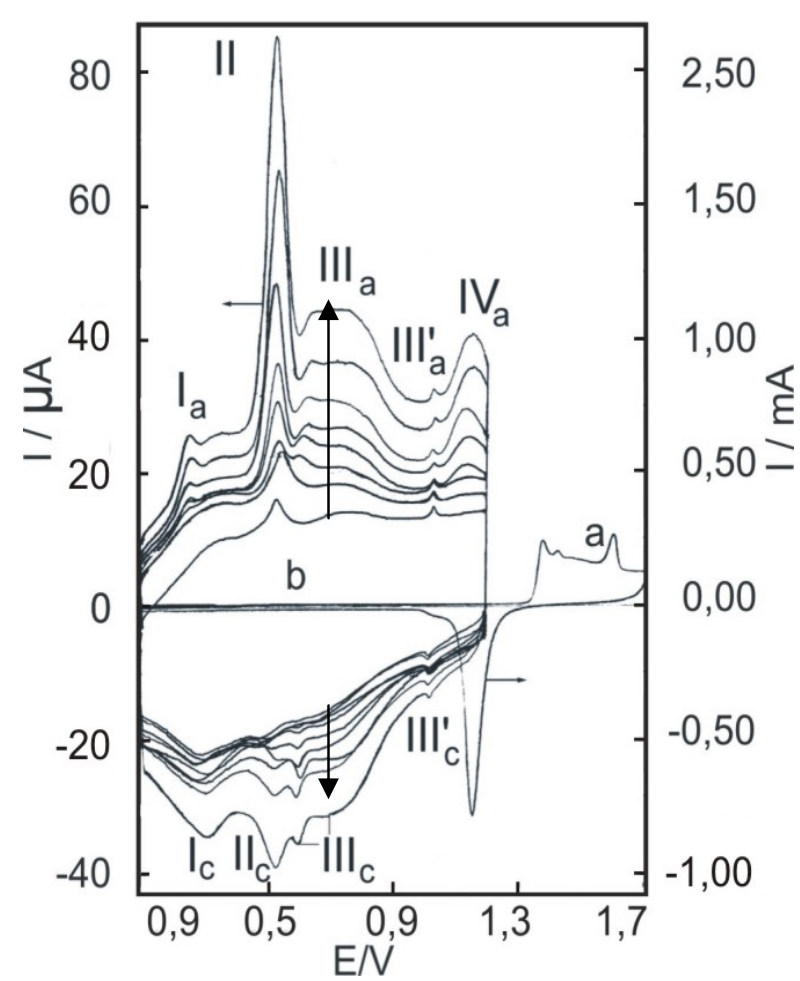

Fig. 3: (a) Voltamperograma del oro con orientación preferida (111) en ácido sulfúrico $0,5 \mathrm{M}$ barriendo en dirección anódica entre 0,1 y 1,7 V. (b) Influencia del tiempo de electrólisis en $0,1 \mathrm{~V}$ sobre los voltamperogramas obtenidos barriendo en dirección anódica entre 0,1 y 1,3 V. $\mathrm{t}=0,10,30,60,120,180,240$ y $300 \mathrm{~min}$ con $t$ aumentando en el sentido señalado por las flechas. $40 \mathrm{mV} / \mathrm{seg} ; 25^{\circ} \mathrm{C}$.

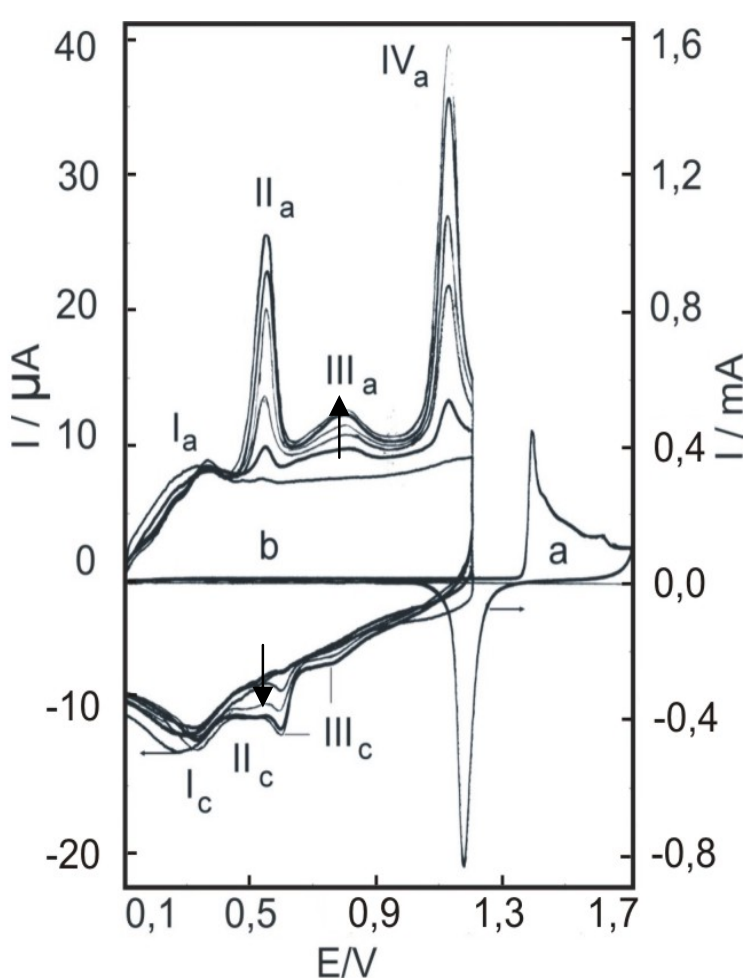

Fig. 4: (a) Voltamperograma del oro con orientación preferida (100) en ácido sulfúrico $0,5 \mathrm{M}$ barriendo en dirección anódica entre 0,1 y 1,7 V. (b) Influencia del tiempo de electrólisis en $0,1 \mathrm{~V}$ sobre los voltamperogramas obtenidos barriendo en dirección anódica entre 0,1 y 1,3 V. $t=0,20,60,120,180$ y 240 min con $t$ aumentando en el sentido señalado por las flechas. $40 \mathrm{mV} / \mathrm{s} ; 25^{\circ} \mathrm{C}$.

La fig. 4, muestra voltamperogramas obtenidos en forma similar a aquellos de la fig. 3 pero con un electrodo orientado según el plano (100). A potenciales bajos, entre 0,1 y $0,3 \vee l_{a}$ decrece con $t$, mientras que a $0,34 \mathrm{~V}$ crece. El pico $I I_{a}$ crece continuamente, pero en menor escala que con el electrodo orientado según el plano (111). Esto es coherente con la menor cantidad de sitios (111) sobre el electrodo orientado según el plano (100). La onda $I I I_{a}$ crece con $t$, alcanzando una altura límite para $t=120$. Es destacable que ese tiempo es más bajo que el mismo para la onda $I I I_{a}$ del electrodo policristalino, y el pico de corriente es relativamente mayor. Esto es lo que habría de esperar para el electrodo orientado según el plano (100): la cantidad de sitios (100) es obviamente mayor. Como la superficie está orientada preferentemente según el plano (100) la PASR tiene lugar preferentemente sobre el plano (100) y, el proceso es más rápido. La velocidad de una reacción depende de la concentración del reactivo. Aquí el reactivo son los sitios (100) de la superficie, cuya concentración ha sido incrementada con el procedimiento de orientación. Y la reacción es la reconstrucción asistida por el potencial (PASR). El pico principal es ahora el pico $I V_{a}$, en ca.1,1 V, que crece continuamente con t. En consecuencia, se lo atribuye a la oxidación de $\mathrm{H}$ sobre el plano (100). A este potencial tan alto es posible el comienzo de la descarga del agua de acuerdo a la reacción:

$(\mathrm{H}) \mathrm{Au}_{(100)}+\mathrm{H}_{2} \mathrm{O} \leftrightarrow(\mathrm{OH}) \mathrm{Au}_{(100)}+2 \mathrm{H}^{+}+2 \mathrm{e}$ 
Según Burke (Burke,1994; Burke y O’Leary, 1989; Burke et al., 1989), la descarga del agua sobre oro policristalino comienza en $0,9 \mathrm{~V}$ (sobre el electrodo policristalino), potencial al cual se forma un óxido incipiente hidratado $(\mathrm{OIH})$. El intermediario en la formación de este óxido es el $\mathrm{OH}$ adsorbido sobre los sitios de baja coordinación. El plano (100) es un plano de baja coordinación y por lo tanto la formación del $(\mathrm{OIH})$ está favorecida. La corriente $I I I_{c}$ tiene dos contribuciones: una onda a 0,75 $\mathrm{V}$ y un pico a $0,60 \mathrm{~V}$ ambos crecen con $\mathrm{t}$ y alcanzan una altura límite a $\mathrm{t}=120$. $I_{c}$ tiende a decrecer con $\mathrm{t}$. Los picos $I_{a}$ y $I V_{a}$ crecen indefinidamente con $t$ (en la práctica se esperaron tiempos de 24 horas). Esto se debe a la acumulación de $\mathrm{H}$ formado sobre dos planos cristalinos distintos a través de los cuales el producto ingresa adentro del metal. La fig. 5 muestra el número de ciclos necesarios para consumir por completo el reactivo depositado sobre los planos (111) y (100). El ciclo en el cual los picos dejan de aparecer, representa al electrodo ya despojado del reactivo. Este ha sido consumido

Tabla 1: Átomos del retículo elemental sobre planos en el sistema cúbico centrado en las caras: $n / a^{2}$ (a=arista).

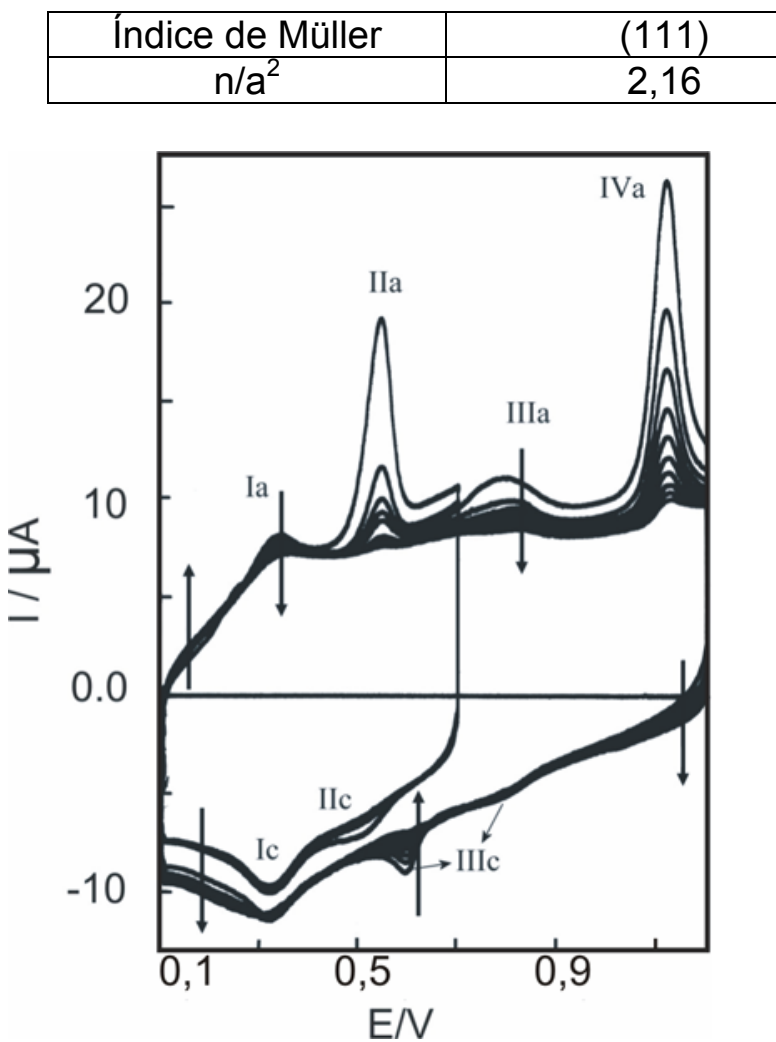

Fig. 5: Voltamperogramas obtenidos en dos etapas con oro orientado según el plano (100) con $t=120^{\prime}$ mostrando el efecto de los ciclos sucesivos. $40 \mathrm{mV} / \mathrm{s} .25^{\circ} \mathrm{C}$.

\begin{tabular}{|c|c|}
\hline$(110)$ & $(100)$ \\
\hline 1,06 & 1,00 \\
\hline
\end{tabular}

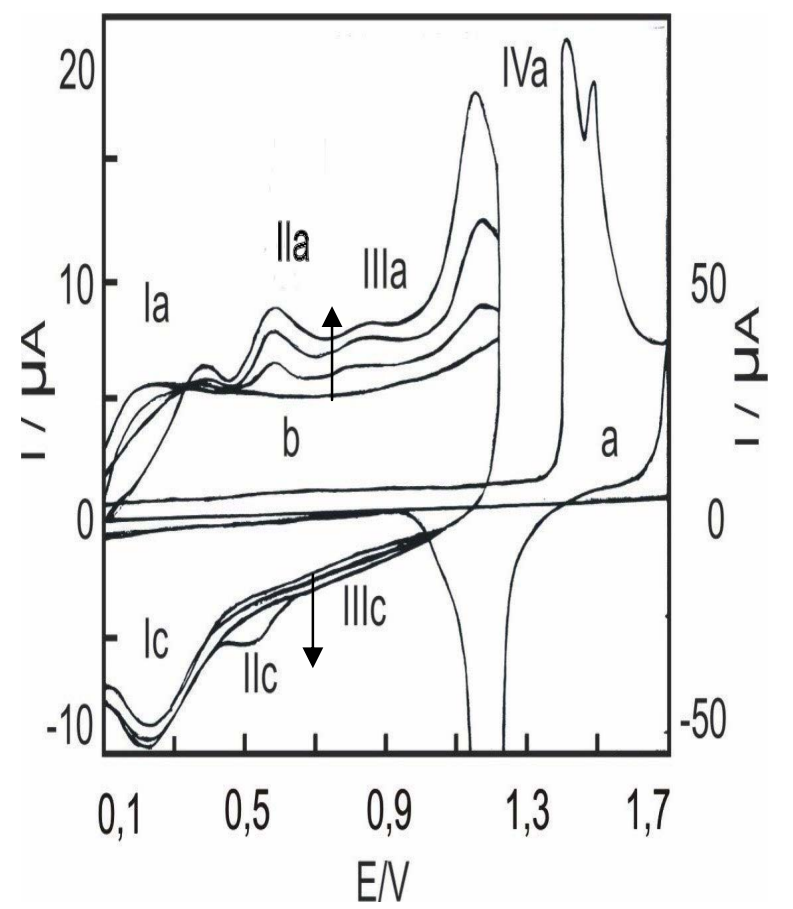

Fig. 6: Voltamperogramas obtenidos con oro orientado según el plano (110) con tiempos de electrólisis a $0,1 \mathrm{~V}$ de $0,20,60$ y 120 min aumentando en el sentido de las flechas. 40 $\mathrm{mV} / \mathrm{s} ; 25^{\circ} \mathrm{C}$.

por oxidación durante los ciclos previos. La experiencia de la figura 5 consiste en dos etapas: La primera etapa consiste en seis ciclos entre 0,1 y $0,7 \mathrm{~V}$. La segunda consiste en diez ciclos entre 0,1 y $1,2 \mathrm{~V}$. No se esperó ningún tiempo entre ambas etapas. De la figura se deduce que el $\mathrm{H}$ formado durante t no ha reaccionado completamente durante el primer ciclo y que hay una cantidad de sustancia formada que reacciona en los ciclos siguientes. Sin embargo, como la corriente disminuye a medida que transcurren los ciclos, se deduce que la sustancia que reaccionó no permanece en la interfase ni se recupera durante el ciclado. El producto, que es el ion $\mathrm{H}^{+}$, difunde hacia la solución, mientras que el reactivo (hidrógeno atómico presente dentro del metal) difunde hacia la superficie donde se oxida hasta consumirse por completo. La fig. 5 muestra la independencia entre los procesos que ocurren en $I_{a}$ y $I V_{a}$ : Es una reacción sobre dos planos distintos: el plano (111) y el plano (100) respectivamente. Después que el reactivo en el plano (111) se consumió en la primera etapa, el reactivo sobre el plano (100) reacciona hasta consumirse en la segunda etapa. Merece destacarse que la 
oxidación de $\mathrm{H}$ ocurre sobre superficies no estructuradas. Sobre una estructura más abierta, como es el caso de la superficie (100), el H necesita mayores potenciales para oxidarse, pues allí está ligado con mayor fuerza.

La fig. 6 muestra los voltamperogramas sobre la superficie orientada según el plano (110). En $I_{a}$ la corriente al principio baja con $\mathrm{t}$, mientras que un pico en $0,38 \mathrm{~V}$ crece, similarmente a lo que ocurría con el electrodo orientado según el plano (100). Sin embargo, en este caso el fenómeno es mucho más claro y más notorio. El pico $I_{a}$ crece con t pero no lo hace en forma tan destacada como en el caso de las orientaciones (111) y (100). El crecimiento más notorio del pico $I I_{a}$ se observó en el caso del electrodo orientado según el plano (111) y por eso que el pico $I_{a}$ se atribuyó a la oxidación del $\mathrm{H}$ sobre este plano. Sobre el electrodo orientado según el plano (110) (fig. 7), la cantidad de sitios (111) es pequeña, pues la superficie ha sido orientada según el plano (110). La onda $I I I_{a}$ no es significativa. Esto es consecuente con el hecho de que la superficie tiene poca cantidad de sitios (100), porque la orientación preferencial es según el plano (110) y III $I_{a}$ se debe al LSR sobre el plano (100). El pico $I V_{a}$ es el mas alto del perfil y su potencial es casi el mismo que en la superficie orientada según el plano (100) (fig.4). El grado de compactación de estos dos planos es muy similar (Tabla 1) y los potenciales para la reacción 2 en ambos planos deben ser muy próximos entre si. Se han realizado barridos voltamperométricos entre 1,35 y $0,80 \mathrm{~V}$ en dirección catódica con velocidades de barrido crecientes, para encontrar una contribución de corriente complementaria con la corriente $I V_{a}$. Efectivamente, se encuentra una onda de corriente catódica en ca.1,07 V (Fig. 7). Para velocidades de barrido crecientes, esta parece desdoblarse en dos, muy próximas entre si, que bien podrían corresponder a la inversa de la reacción (2) sobre los planos (110) y (100). La figura 8b muestra voltamperogramas obtenidos con oro con orientación (100) para t entre 1 y 60 minutos. Se realizó con escala más sensible que la de la figura 4 y se observa que el pico $I V_{a}$ también se desdobla en dos. Según Kolb, el plano (110) adquiere la configuración (1x2), reconstrucción asistida por el potencial. EI LSR ocurre a potenciales bajos asistida por el potencial y no se manifiesta en los voltamperogramas. En este trabajo no se encuentra onda o pico atribuíble a ese fenómeno, resultado que concuerda con Kolb.

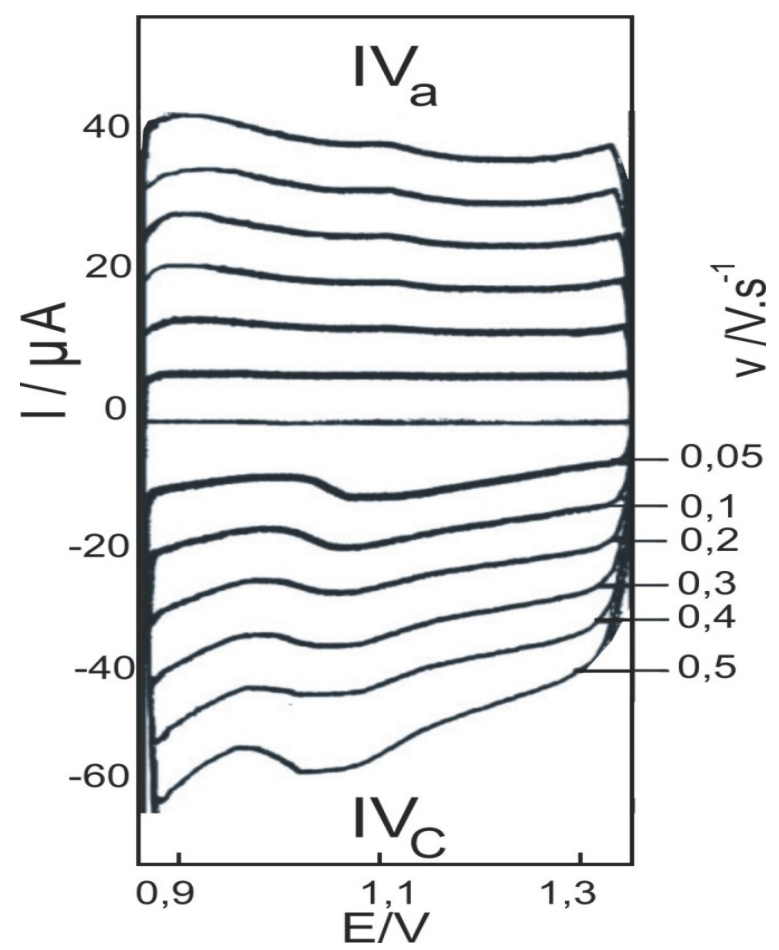

Fig. 7 (derecha) Voltamperogramas obtenidos con el oro policristalino barriendo en dirección catódica entre 1,35 y $0,80 \mathrm{~V}$ con diferentes velocidades de barrido. $25^{\circ} \mathrm{C}$

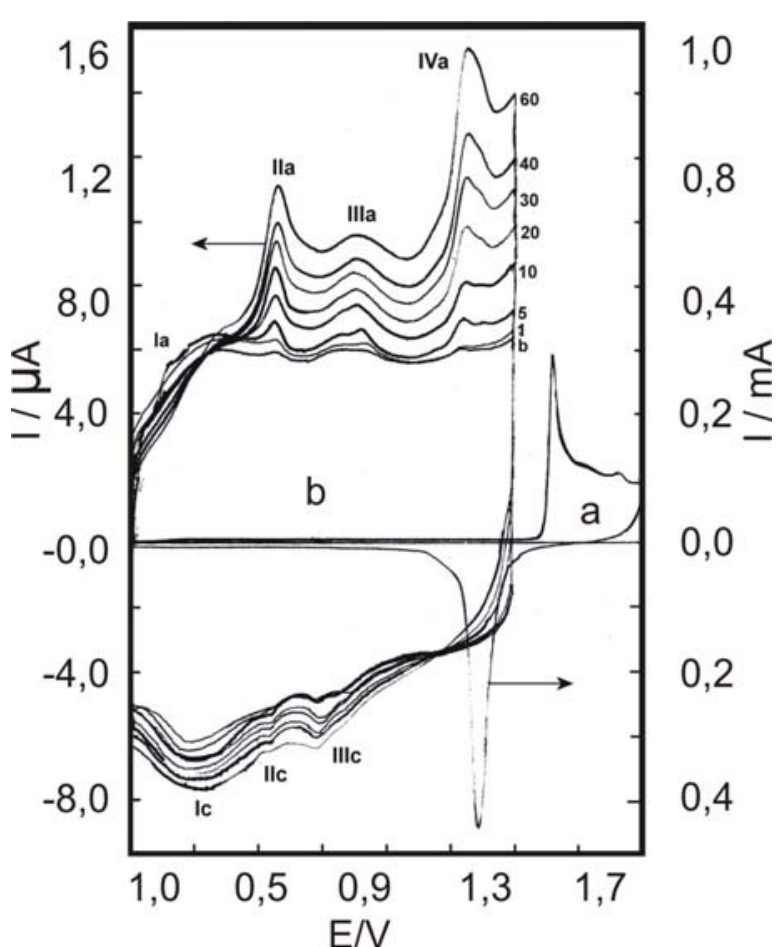

Fig.8 (izquierda) Voltamperogramas obtenidos con electrodo de oro con orientación preferida (100) con tiempos de electrólisis de $0,1,5,10$, $2030,40,60 \mathrm{~min} .40 \mathrm{mV} / \mathrm{s} .25^{\circ} \mathrm{C}$.

En todos los experimentos descriptos hasta aquí, el burbujeo de nitrógeno se detenía durante la corrida voltamétrica. En algunos casos esto no se hacía y la solución tenía agitación durante la experiencia. En estos casos, la agitación de la solución no cambiaba la corriente en el primer ciclo, pero 
causaba la desaparición de los ciclos subsiguientes. La corriente del primer ciclo era la misma sin y con agitación. Los ciclos siguientes no pudieron sobrevivir en la solución agitada. Evidentemente, hay una etapa de transferencia de masa participando en el proceso: $\mathrm{H}$ molecular resulta de la etapa de recombinación de $\mathrm{H}$ atómico y se remueve por difusión convectiva de la solución. En el primer ciclo reacciona el $\mathrm{H}$ atómico adsorbido. Este no puede ser removido por la agitación de la solución ción. Se trata de $\mathrm{H}$ adsorbido fuertemente. En condiciones ordinarias, el $\mathrm{H}$ atómico superficial está presente en muy pequeñas cantidades. Pero, hay condiciones excepcionales, cuando las capas metálicas cerca de la superficie se cargan con $\mathrm{H}$ atómico. El $\mathrm{H}$ en el interior estabiliza el $\mathrm{H}$ adsorbido, porque el gradiente de concentración que posibilita la difusión de $\mathrm{H}$ hacia adentro, cae cuando la concentración de $\mathrm{H}$ es alta cerca de la superficie. Cuando el potencial químico del $\mathrm{H}$ en la zona interna cercana a la superficie es alto, la posterior difusión hacia adentro se ve impedida y esto favorece la adsorción. Este fenómeno ha sido desconocido hasta el presente.

Con todas las superficies estudiadas aquí, tiempos largos conducen a mayores corrientes de LSR Cuando la reconstrucción es completa se alcanza una altura límite. La corriente $I I I_{a}$ crece con t pero alcanza un valor límite a 180' para la superficie policristalina y 120' para la superficie (111). Con la superficie (111) el tiempo límite no se muestra por el efecto de enmascaramiento debido a la gran altura del pico $I I_{a}$. Este tiempo límite es mucho más largo que el utilizado por Kolb (1996) para alcanzar una altura límite del pico debido al LSR sobre monocristales. Pero la cinética de Kolb del PASR sobre monocristales, debe ser diferente a aquella sobre electrodos policristalinos. La presencia de $\mathrm{H}$ modifica la cinética del PASR. Sobre monocristales el pcc está localizado a un potencial alejado en dirección anódica de aquel correspondiente a la formación de H (Kolb, 1996) y así es posible cargar negativamente el metal sin cargarlo con $\mathrm{H}$. La policristalinidad de la superficie desplaza la reacción de deposición de $\mathrm{H}$ hacia valores más positivos, y por eso no es posible cargar negativamente el metal, sin que ocurra la descarga de $\mathrm{H}$. Evidentemente los bordes de grano juegan un papel importante en la deposición de $\mathrm{H}$. Se supone que la primera etapa de la reacción ocurre sobre los bordes de grano. Luego, el hidrogeno atómico difunde sobre la superficie y al mismo tiempo difunde hacia el interior del grano. Chao no encontró difusión de $\mathrm{H}$ a lo largo de los bordes del grano (Chao et al., 1980). Pero es probable que la alta concentración de defectos existentes cerca del borde de grano catalice la deposición de $\mathrm{H}$ y esta es la razón de que el sobrepotencial de la deposición de $\mathrm{H}$ es menor que la del electrodo policristalino comparado con aquel de la superficie monocristalina.

Los picos $I_{c}$ y $I I I_{c}$ se definen mejor con $v$ mayores (fig.2) y el pico $I_{c}$ muestra dos contribuciones de corriente debidas al cambio de la capacidad de la doble capa por la desorción de aniones en las superficies (111) y (110) no reconstruidas. La corriente $I I I_{c}$ se debe a la desorción de aniones en el plano (100) no reconstruído. $I I I_{c}$ puede tener también la contribución del óxido de oro hidratado formado simultáneamente con la formación de $\mathrm{H}$ en $I V_{a}$ (Burke, 1994). Esto explicaría el crecimiento de $I I I_{c}$ con $\mathrm{t}$ (fig.1). Si no se barre el potencial de $I V_{c}$ la corriente $I I I_{c}$ no crece con $t$ (Sustersic et al., 2004).

Es posible obtener picos $\| I_{a}$ muy altos cargando el electrodo con $\mathrm{H}$ mediante electrólisis a potenciales catódicos. Pero el pico $I_{c}$ se puede incrementar solo un poco por crecimiento de la concentración local del ión $\mathrm{H}$ en la reacción que ocurre en $I_{a}$. El potencial de los picos $\|_{a}$ y $\|_{c}$ es casi el mismo. Este es el requerimiento de una reacción sumamente reversible. Sin embargo la corriente anódica de $\|_{a}$ no tiene el mismo valor absoluto que la corriente catódica de $I_{c}$. La razón es que la fuente del reactivo es distinta. En el caso del pico $I_{a}$ el $\mathrm{H}$ proviene del interior del metal, mientras que en el caso del pico $I_{c}$ el reactivo es el ión $\mathrm{H}^{+}$presente en el electrolito. La concentración del ión $\mathrm{H}^{+}$aumenta cerca del electrodo cuando ocurre la oxidación de $\mathrm{H}$ en $I_{a}$ y por lo tanto la corriente en $I_{c}$ crece en el ciclo catódico a medida que crece el pico $I_{a}$. Sin embargo, una parte del ión $\mathrm{H}^{+}$formado en $I_{a}$ difunde hacia el seno del electrolito. La influencia del transporte de materia en la reacción que ocurre en $I_{a}$ es evidente, como se observa en la fig. 5 que muestra la reacción de oxidación de $\mathrm{H}$ en dos planos cristalinos distintos: (111) y (100) respectivamente. El experimento de la fig.5 se hizo con una superficie preferentemente orientada según el plano (100). El mismo efecto se observa con las superficies orientadas según los otros planos de bajo índice (las figuras no se muestran aquí).

La corriente en $I V_{a}$ tiene las contribuciones de la oxidación de $\mathrm{H}$ sobre los planos (100) y (110) y de la formación del óxido incipiente hidratado (Burke,1994; Burke y O’Leary, 1989; Burke et al., 1989). 


\section{CONCLUSIONES}

A partir de los resultados obtenidos se concluye que el perfil potenciodinámico del oro policristalino en la región de potenciales de carga de doble capa no presenta sólo contribuciones de corriente capacitivas debidas a la carga de doble capa y a los cambios en la capacidad de esta por causa de la adsorción y desorción de aniones y al levantamiento de la reconstrucción superficial de los diferentes planos cristalinos que la superficie expone al electrolito, sino también presenta contribuciones farádicas originadas por el sistema redox del hidrógeno. Este presenta reversibilidad en cuanto al potencial, pero los valores absolutos de las corrientes de reducción y oxidación son distintos pues las fuentes del reactivo son diferentes: en el caso de la oxidación, el reactivo es hidrógeno atómico que difunde desde el interior del metal. En el caso de la reducción, el ion $\mathrm{H}^{+}$se encuentra disuelto en el electrolito. La recombinación de los átomos en la superficie y el transporte de materia juegan un papel fundamental en la reacción redox. El papel del transporte de materia se manifiesta cuando se produce la agitación del electrolito. En ese caso permanecen sobre la superficie sólo aquéllos átomos que lograron la adsorción. Cuando la concentración de $\mathrm{H}$ en el interior del metal cercana a la superficie es alta, la posterior difusión hacia adentro se ve impedida y la adsorción se ve favorecida. La contribuciones de corriente de LSR se distinguen de las contribuciones farádicas porque al aumentar $t$ alcanzan una altura límita, mientras que aquéllas de oxidación de hidrógeno continúan creciendo a medida que aumenta la cantidad de $\mathrm{H}$ que se carga durante la electrólisis catódica.

A continuación se presenta un resúmen de las contribuciones de corriente que se encuentran cuando se analiza la estructura fina del voltamperograma obtenido con escala sensible en la región de potenciales de carga de doble capa:

$l_{a}$ ) los cambios en la capacidad de la doble capa producidos por la adsorción de aniones y al LSR del plano (111). $I_{c}$ ) los cambios de capacidad debidos a la desorción de aniones. $I_{a}$ ) la oxidación del $\mathrm{H}$ atómico que difunde dede el interior del metal sobre el plano (111) no reconstruido. $I I_{c}$ ) la (HUPD) sobre el plano (111) que resulta en una pequeña cantidad de $\mathrm{H}$ adsorbido. III ) LSR del plano (100) y el cambio en la capacidad de la doble capa debido al reacomodamiento de los iones adsorbidos sobre el plano (100) no reconstruido. $I I I_{c}$ ) la desorción de aniones en el plano (100) y la PASR parcial del plano (100) y/o la reducción del óxido incipiente hidratado. $I V_{a}$ ) la oxidación del H atómico sobre los planos (100) y (110) (el H difunde desde el interior del metal)(reacción 2) y la posible formación del óxido incipiente hidratado. $I V_{c}$ ) La inversa de la $I V_{a}$ (inversa de la reacción 2).

\section{AGRADECIMIENTOS}

Este trabajo ha sido subsidiado por la Universidad Nacional de San Luis. M.G. Sustersic es miembro del Consejo Nacional de Investigaciones Científicas y Técnicas.

\section{REFERENCIAS}

Burke, L.D. y W.A. O'Leary; "The importance of superficial (adatom) surface oxidation in the electrocatalytic behaviour of noble metals in aqueous media" J. of Applied Electrochemistry: 19, 758-767 (1989)

Burke, L.D., J.F. Healy, K.J. O`Dwyer y W.A. O`Learly; "Incipient Hydrous Oxides-The Missing Link in Noble Metal Electrocatalysis” J. Electrochem.: Soc. 136(4), 1015-1021 (1989).

Burke, L.D.; "Premonolayer oxidation and its role in electrocatalysis" Electrochim. Acta: 39(11/12), $1841-1848(1994)$

Chao, F., M. Costa y R. Parsons; "SIMS analysis of the distribution of Hydrogen absorbed by gold electrodes during the evolution of Hydrogen" J. Electroanal. Chem.: 115, 31-44 (1980).

Córdova Orellana, R.C., M.E. Martins y A.J. Arvía; "Potentiodynamic oxidation of Absorbed and adsorbed Hydrogen atoms on gold promoted by the dynamic ageing of the oxygen containing layer. Electrochimica Acta: 24, 469-471 (1979). 
Florit, M.I., M.E. Martins y A.J. Arvía; "La influencia de los aniones em la electroadsorción de oxigeno sobre oro policristalino em médio ácido". An. Asoc. Quim. Argent.: 72, 71-92 (1984).

Jaram, R., A.Q. Contractor y Lal. Hira; "Formic Acid Oxidation at Platinized Platinum Electrodes. Part IV. Effect of Preadsorbed Sulfur and Related Studies: J. Electroanal. Chem. 87, 225-237 (1978).

Kolb, D.M.; "Reconstruction phenomena at metal-electrolyte interfaces". Progress in Surface Science: 51(2), 109-173 (1996).

Kolb, D.M.; "Surface Reconstruction at Metal-Electrolyte Interfaces" Cap. 3 in: "Structure of Electrified Interfaces”, Lipkowski J. and Ross P. N. (Eds.).VCH, New York p. 65-102 (1993).

Kunning, A.E. Von M. y otros cuatro autores; "Estudio electroquímico de la adsorción del $\mathrm{SO}_{2}$ sobre oro. An. Asoc. Quím. Argent.: 74(4), 345-351 (1986).

Martins, M.E., J.J. Podestá y A.J. Arvía; "Chemical evidence of Hydrogen sorption processes on potential cycled gold electrodes”. Electrochim. Acta: 32(7), 1013-1017 (1987).

Perdriel, C.L., A.J. Arvía y M. Ipohorski; "Electrochemical Faceting of polycrystalline gold in $1 \mathrm{M}$ $\mathrm{H}_{2} \mathrm{SO}_{4}$ ". J. Electroanal. Chemistry: 215, 317- 329 (1986)

Rand, D.A.J. y R. Woods; "A Study of the Dissolution of Platinum, Palladium, Rhodium and Gold Electrodes in $1 \mathrm{M}$ Sulphuric Acid by Cyclic Voltammetry" Electroanalytical Chemistry and Interfacial Electrochemistry: 35, 209-218 (1972).

Schmid, G.M.; "Hydrogen Overvoltage on Gold”, Electrochim. Acta: 12, 449-459 (1967)

Schneider, J. y D.M Kolb; "Potential induced surface reconstruction of Au(100)". Surface Science: 193, 579-592 (1988).

Shi, Z. y otros cuatro autores; "Investigations of sulfate ion adsorption at the $\mathrm{Au}(111)$ electrode by chronocoulometry and radiochemistry" J. Of Electroanalytical Chemistry: 366 317-326 (1994).

Shi, Z., J. Lipkowski, S. Mirwald y B. Pettinger; "Electrochemical and second harmonic generation study of sulfate adsorption at Au(111) electrode" J. Of Electroanalytical Chemistry: 396, 115-124 (1995).

Sustersic, M.G., D.E. Moreno, N.V. Almeida y M. Ipohorski; "Técnica Potenciodinámica para cambiar la Morfología Superficial del Oro”, Inf. Tecnol.: 13(2), 75- 80 (2002).

Sustersic, M.G., D. Moreno y A.E. Von Mengershausen; “La deposición a subpotencial de Hidrógeno sobre Oro policristalino". Inf. Tecnol.; 15(4), 85-90 (2004).

Sustersic, M.G. y otros seis autores; "La dependencia con el tiempo de la adsorción del $\mathrm{SO}_{2}$ sobre platino", An. Asoc. Quim. Argent.: 77(3) 277-286 (1989).

Von Mengershausen, A.E., J.A. Quiroga y M.G. Sustersic; "La influencia del pretratamiento del electrodo de platino sobre la adsorción del $\mathrm{SO}_{2}$ ", An. Asoc. Quim. Argent.: 77(3) 265-276 (1989).

Vela, M.E., S.L. Marchiano, R.C. Salvarezza y A.J. Arvía; "Growth of columnar gold electrodes by electroreducing hydrous gold oxide layers: kinetics and mechanistic interpretation", J. of Electroanlytical Chemistry 388, 133-141 (1995).

Zaniolo, S.M., C. Abaca y M.G. Sustersic; "La reducción de $\mathrm{SO}_{2}$ sobre platino con formación de un catalizador superficial”, An. Asoc. Quim. Argent.: 84, 552(1996). 
